\title{
Magnetic circular dichroism in the ion yield of polarized chromium atoms at the $2 p$ edge
}

\author{
G. Prümper, ${ }^{1}$ S. Kröger, ${ }^{2}$ R. Müller, ${ }^{2}$ M. Martins, ${ }^{3}$ J. Viefhaus, ${ }^{1}$ P. Zimmermann, ${ }^{2}$ and U. Becker ${ }^{1}$ \\ ${ }^{1}$ Fritz-Haber-Institut der Max-Planck-Gesellschaft, Faradayweg 4-6, D-14195 Berlin, Germany \\ ${ }^{2}$ Institut für Atomare Physik und Fachdidaktik, Technische Universität Berlin, Hardenbergstrasse 36, D-10623 Berlin, Germany \\ ${ }^{3}$ Institut für Experimentalphysik, Universität Hamburg, Luruper Chaussee 149, D-22761 Hamburg, Germany
}

(Received 9 May 2003; published 12 September 2003)

\begin{abstract}
The effect of magnetic dichroism in the partial and total ion yield of chromium, i.e., the absorption of polarized chromium vapor was observed in the gas phase. The measurements were performed at the $2 p$ edge and at photon energies above the $2 p$ edge. The structure of the dichroism at the $2 p$ edge can be understood by including the coupling of the $2 p$ hole with the $3 d$ and $4 s$ shells. Our experimental results for the dichroism at the $2 p$ edge are similar to results of solid-state experiments. Implications for the sum rules used as a standard tool to calculate the spin and orbital momentum are discussed.
\end{abstract}

DOI: 10.1103/PhysRevA.68.032710

PACS number(s): 32.80.Fb, 32.70.Fw

\section{INTRODUCTION}

Magnetic dichroism describes the different responses of a magnetized sample to differently polarized light. Many solidstate measurements of dichroism effects were performed on transition-metal samples. Due to the difficulties of the evaporation and polarization of the transition metals there are only few dichroism experiments in the gas phase. Unlike other transition metals chromium can be polarized in its ground state by laser pumping without frequency doubling. A number of electron spectra could be measured on polarized chromium [1-5]. However, for a direct comparison with solidstate experiments, i.e., with absorption measurements of thin magnetic films, an absorption or total-ion-yield measurement is needed. Ion-yield gas phase experiments have so far only been performed with unpolarized $\mathrm{Cr}$ atoms [6] although the experimental technique was already used in the early 1970s for polarized alkali atoms $[7,8]$.

Magnetic circular dichroism (MCD) describes the different responses of a magnetized sample to left- and righthanded circularly polarized light and is expected to show the most pronounced dichroism effects of all absorption measurements. In this work we present the first MCD measurements in the total and partial ion yield of atomic chromium. Neglecting fluorescence, the total ion yield is equivalent to the absorption but unlike a real absorption measurement an ion-yield measurement can resolve the different charge states of the ions created by different decay paths. Thus an ionyield experiment can compare the dichroism of the differently charged ions and test the validity of the two-step model, which separates the process into excitation and decays. As only the excitation process is expected to depend on the helicity of the light the dichroism should not depend on the ions charge. Additionally the absence of dichroism above the $2 p$ edge can be tested with high accuracy. A nonresonant MCD effect in the ion yield was found for iron atoms in an earlier experiment [9]. For chromium nonresonant MCD can only be caused by relativistic effects, as the ground state of chromium has no orbital angular momentum [10].

The quantity commonly used to express the relative strength of the MCD is the MCD asymmetry:

$$
(\text { asymmetry })=\frac{I_{\uparrow \uparrow}-I_{\downarrow \uparrow}}{I_{\uparrow \uparrow}+I_{\downarrow \uparrow}} .
$$

$I_{\uparrow \uparrow}$ and $I_{\downarrow \uparrow}$ are the measured ion yields or absorption cross sections for photon spin and atomic magnetization parallel and antiparallel to each other, respectively.

Independent of the complicated excitation dynamics near the $2 p$ edge, sum rules for the MCD asymmetry have been used to determine the expectation value of the orbital and spin contributions to the magnetic dipole momentum $m_{\text {orb }}$ and $m_{\text {spin }}$ [11-13] of initial state for different transitionmetal samples. These sum rules, based on single-particle models not taking into account the many-body character of the core hole excited state involve integration of the cross sections over selected intervals of the resonant region and over the complete $2 p$ edge:

$$
\begin{gathered}
m_{o r b} \propto-\frac{\int_{L_{3}+L_{2}}\left(\mu_{+}-\mu_{-}\right) d \omega}{\int_{L_{3}+L_{2}}\left(\mu_{+}+\mu_{-}\right) d \omega}, \\
m_{\text {spin }} \propto-\frac{6 \int_{L_{3}}\left(\mu_{+}-\mu_{-}\right) d \omega-4 \int_{L_{3}+L_{2}}\left(\mu_{+}-\mu_{-}\right) d \omega}{\int_{L_{3}+L_{2}}\left(\mu_{+}+\mu_{-}\right) d \omega},
\end{gathered}
$$

where $\mu_{-}$and $\mu_{+}$are the absorption cross sections for the two different target polarizations, i.e., magnetization directions for thin films. They can be replaced with the ion count rates $I_{\uparrow \uparrow}$ and $I_{\downarrow \uparrow}$ measured in our experiment. $L_{3}$ and $L_{2}$ denote the integration ranges. The use of these indices implies that the $2 p$ edge can be divided into two intervals each containing only $L_{2}$ or $L_{3}$ contributions. Equation (2) can be used without any problem as the integration range extends over the whole $2 p$ edge and the choice of the integration range is not critical. Of course $m_{\text {orb }}$ must be zero for the $\mathrm{Cr}$ ground state $[\mathrm{Ar}] 3 d^{5} 4 s^{17} S_{3}$. Equation (3) contains an integral over the $p_{1 / 2}$ states only. We examined the structure of 
the $2 p$ edge for the chromium atom and will discuss the problem of choosing the region $L_{3}$ later.

\section{PROCEDURE}

Chromium is heated in an electron-impact oven to a temperature of about $1800 \mathrm{~K}$. The atoms become polarized by laser pumping when they pass a circularly polarized laser beam. The laser system will be described in more detail below. A magnetic field of $B \approx 500 \mu \mathrm{T}$ parallel to the propagation direction of the laser was applied to avoid any uncontrolled loss of target polarization by rotation of the magnetic moment around the magnetic field. The laser was $2 \mathrm{~mm}$ closer to the oven than the ionizing synchrotron radiation to make sure that no excited atoms, created during the pumping process, contribute to the target.

The beam of polarized chromium atoms crosses the beam of circularly polarized vacuum ultraviolet synchrotron radiation from a Sasaki-type undulator [14]. The experiment was performed at the UE56-2 PGM1 beam line at BESSY II synchrotron light source [15]. Typical operation parameters were: $h \nu=550$ to $600 \mathrm{eV}$, circular polarization $\left|S_{3}\right|>0.80$, and flux $10^{11}$ photons/s.

The ions were detected in a pulsed time-of-flight ion spectrometer. The operation parameters were: Extraction pulse amplitude approximately $100 \mathrm{~V}$, pulse width $10 \mu \mathrm{s}$, repetition rate $20 \mathrm{kHz}$, and length of the ion path $200 \mathrm{~mm}$. Figure 1 shows the ion time-of-flight spectrum. It contains contributions from unpolarized gas molecules $\mathrm{N}^{+}, \mathrm{O}^{+}, \mathrm{OH}^{+}$and the $\mathrm{Cr}^{n+}$ signal. $\mathrm{N}_{2}$ was introduced into the vacuum chamber during the measurement for calibrating purpose. During a measurement the intensity of the synchrotron radiation varies, as the ring current decreases and the monochromator is used to change the photon energy and thus changes in the beam line transmission are unavoidable. The method to take into account all of these effects at the same time is the use of $\mathrm{N}^{+}$as a reference signal that does not have any resonance in the cross section and no dichroism. By normalizing all $\mathrm{Cr}^{n+}$-count rates to the $\mathrm{N}^{+}$-count rate all sources of error mentioned above are eliminated. In the experiment the different charge states of chromium $\mathrm{Cr}^{+}, \mathrm{Cr}^{2+}, \mathrm{Cr}^{3+}, \mathrm{Cr}^{4+}$, and $\mathrm{Cr}^{5+}$ were observed. The backgrounds in Fig. 1 are caused by ions that are produced while the high-voltage pulse is already switched on at the time-of-flight spectrometer and thus they appear as tails after the peaks that belong to the different masses. This type of background can be fitted easily using only one free parameter (the peak-to-tail ratio). All count rates and asymmetries in this paper refer to the signals after background subtraction.

During the measurement the laser polarization, i.e., the direction of the target polarization was switched with a frequency of $0.1 \mathrm{~Hz}$. The linearly polarized laser is sent through a fresnel rhombus. When the rhombus is rotated by a stepping motor, the polarization plane of the light is tilted by $90^{\circ}$. A $\lambda / 4$ plate behind the rhombus transforms the linear polarization to circular polarization of either positive or negative helicity. During the turning of the rhombus the laser beam is blocked and the chromium atoms remain unpolarized. Depending on the state of the pumping beam the time-
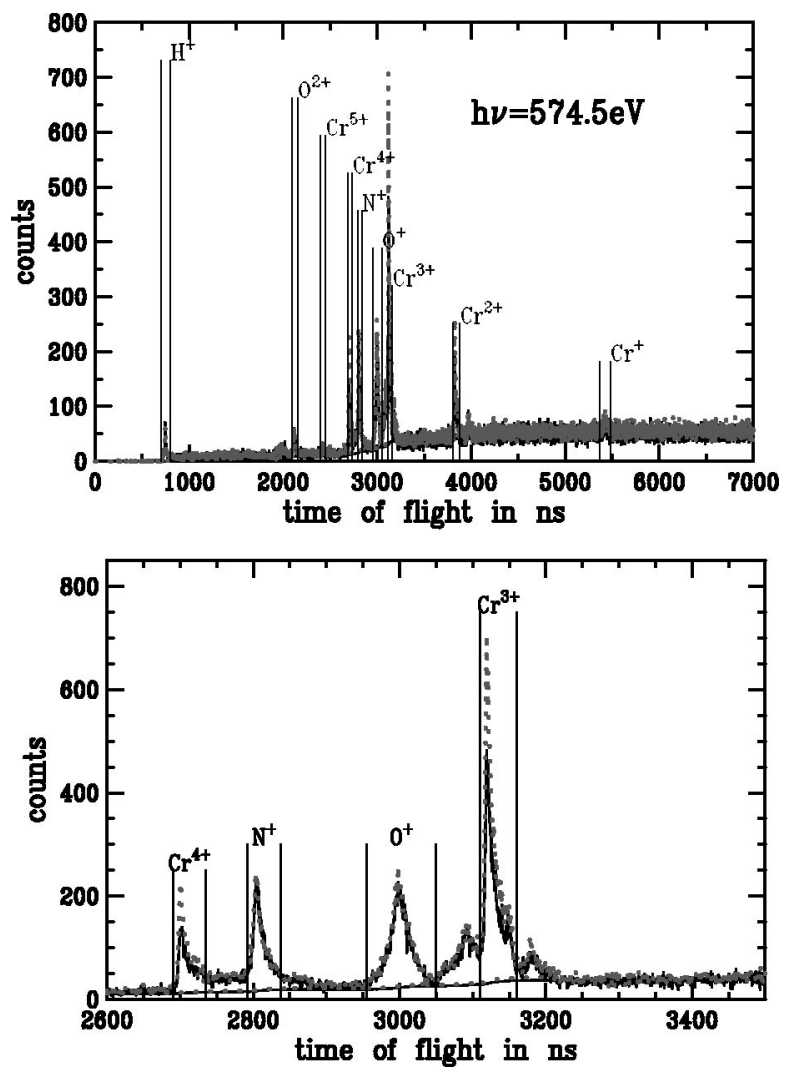

FIG. 1. Ion time-of-flight spectrum. The upper picture shows the complete mass spectrum, the lower picture is a blow up of the mass region 12-20 amu. The solid line belongs to the count rate of one fixed target polarization and the broken line belongs to the count rate of the opposite target polarization. The count rates of the $\mathrm{N}^{+}$ and $\mathrm{O}^{+}$signals do not react to the change of the pump-laser polarization direction, while the $\mathrm{Cr}^{4+}$ and $\mathrm{Cr}^{3+}$ signals clearly do.

of-flight signal of the ions is sent to one out of three input channels of a TDC module. In this way the three measurements of the ion count rate were performed at almost the same time and the measured asymmetry is not influenced by the varying intensities of the synchrotron light source and the atomic beam. The three ion count rates belong to the cases: (A) Circular polarization $S_{3}>0.80$, (B) $S_{3}<-0.80$, and (C) Laser beam blocked $S_{3}=0$. The third measurement $\mathrm{C}$ with the laser blocked is not redundant. The condition $\frac{1}{2}\left(I_{A}+I_{B}\right)$ $=I_{C}$ is only fulfilled if the contribution of the parameter $\beta_{202}$ defined in Ref. [16] can be neglected. We did not observe any deviations from this condition. In order to double check the reliability of the measurement it was repeated with the inverse helicity of the synchrotron light.

\section{LASER SYSTEM AND TARGET POLARIZATION}

The laser system consists of a 5 W UV Argon-ion pump laser and a tunable single-mode $\mathrm{cw}$ dye ring laser with internal frequency locking. The ring laser is run with Stilbene 3. The wavelength is measured with a wavemeter. Additionally we measured the fluorescence light of the corresponding transition in a second separate chromium atomic beam to make sure that the laser did not drift away from the transi- 

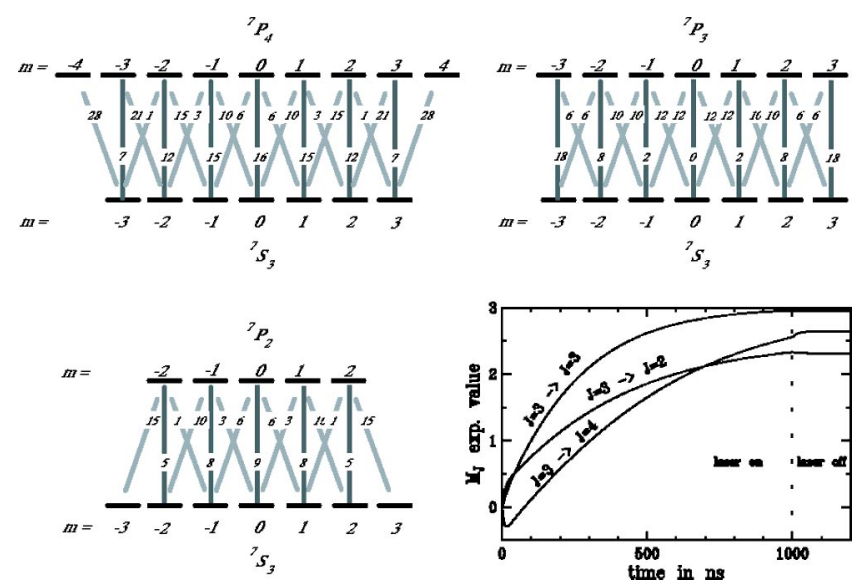

FIG. 2. The transition coefficients for the different excited states used for laser pumping. The diagram in the lower right shows the solutions of the rate equations. The expectation value of $M_{J_{1}}$ for the atoms in the ground state is plotted as a function of time.

tion. The time-averaged flux was $10 \mathrm{~mW}$, the diameter of the beam $\approx 1.5 \mathrm{~mm} \times 1.5 \mathrm{~mm}$, and the bandwidth $\approx 10 \mathrm{MHz}$.

The degree of the polarization of the target can be calculated by solving the rate equations for the known values of the laser intensity and the time of flight of the atoms through the laser beam. By using the asymmetry at $574.6 \mathrm{eV}$ as a reference signal we found that the presence of the guiding magnetic field is important to get maximum polarization of the target. Its strength is not very critical nor is the distance of the laser to the synchrotron radiation. We tried the three chromium transitions $3 d^{5} 4 s^{7} S_{3} \rightarrow 3 d^{5} 4 p^{7} P_{2,3,4}$ : $425.4332 \mathrm{~nm}, E_{\mathrm{u}}=23498.84 \mathrm{~cm}^{-1}, J_{\mathrm{u}}=4 ; 427.4796 \mathrm{~nm}$, $E_{\mathrm{u}}=23386.35 \mathrm{~cm}^{-1}, \quad J_{\mathrm{u}}=3 ; \quad$ and $428.9716 \mathrm{~nm}, \quad E_{\mathrm{u}}$ $=23305.01 \mathrm{~cm}^{-1}, J_{\mathrm{u}}=2$, where $E_{\mathrm{u}}$ is the energy and $J_{\mathrm{u}}$ the total electron angular momentum of the upper level. All three excited states have a spontaneous decay rate of $A_{21}$ $\approx 3 \times 10^{7} \mathrm{~Hz}$. We found the line at $427.4796 \mathrm{~nm}$ to be most efficient. This is consistent with the solution for the rate equations for the experimental conditions (see Fig. 2). It is assumed that each atom is $1 \mu \mathrm{s}$ in the laser light. After that all excited atoms decay to the ground state. The numbers used for this calculation were: Laser output power of $5 \mathrm{~mW}$ $(5 \mathrm{~mW}$ instead of $10 \mathrm{~mW}$ were used in the calculation because of the possible short-time variations of the laser intensity), a beam cross section of $1.5 \mathrm{~mm} \times 1.5 \mathrm{~mm}$ and a bandwidth of $10 \mathrm{MHz}$. The density in the chromium beam was sufficiently low. Thus depolarization due to collisions can be neglected. The calculation yields that the transition $3 d^{5} 4 s^{7} S_{3} \rightarrow 3 d^{5} 4 p^{7} P_{3}$ is suited best for the polarization of the chromium atoms. This is consistent with our experimental results. It turns out that the coefficients shown in the upper right diagram of Fig. 2 are suited best for this transition. At this wavelength a reduction of the laser intensity down to $2.5 \mathrm{~mW}$ was possible without loss of asymmetry in the ion count rates. Even though this suggests that the pumping process reached saturation we get the best agreement between theory and experiment for the scan over the $2 p$ edge if we assume a target polarization of only $40 \%$. This is not in agreement with our model of the pumping process. We either

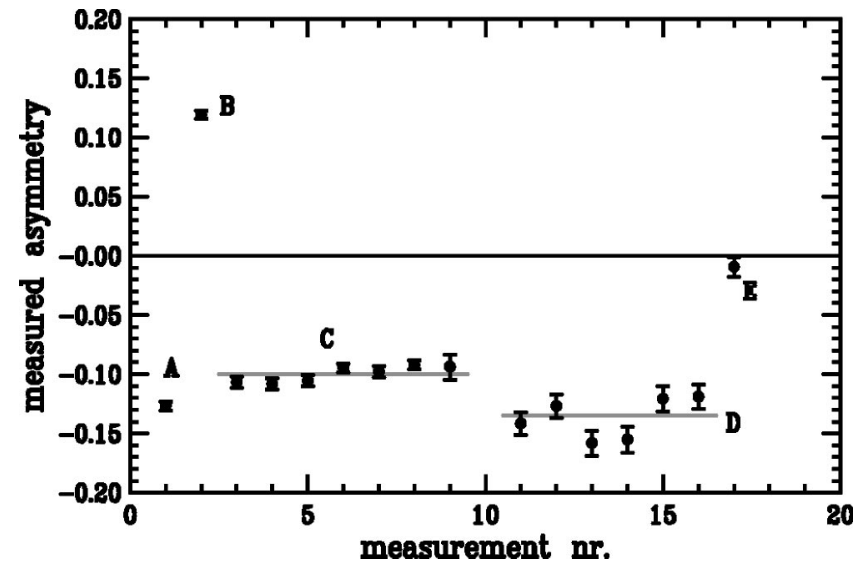

FIG. 3. Study of the long term stability and reproducibility of the asymmetry. All measurements were made at a photon energy of $h \nu=574.6 \mathrm{eV}$. All data points represent the asymmetry of the $\mathrm{Cr}^{3+}$ signal normalized to the $\mathrm{N}^{+}$signal. The points $A$ and $B$ were measured with synchrotron light of opposite helicity. The series $C$ was measured one day later with the same helicity as in case $A$. The differences in the absolute values of the measured asymmetry are attributed to long term changes of the laser intensity or alignment. Series $D$ was measured with reduced output slit width of the monochromator, i.e., with higher photon energy resolution. Point $E$ was measured with the laser switched off.

have to assume that the theoretical values for $\beta_{101}$ are too large or that our description of the pumping process is not appropriate.

\section{PRECISION OF THE MEASUREMENT}

All error bars given in this work are purely statistical error bars $( \pm \sigma)$. In order to test the reliability of our method we repeated the measurement of the asymmetry at a photon energy of $h \nu=574.6 \mathrm{eV}$ many times. Figure 3 shows the result of this study. The measured asymmetry changes sign when the helicity of the ionizing synchrotron radiation is inverted but does not change its size. This is shown by the data points $A$ and $B$ in Fig. 3. The reproducibility of the asymmetry measurement in time intervals of some hours is reasonably good. This is shown in the series $C$ and $D$ in Fig. 3. Series $D$ was measured with reduced output slit width of the monochromator, i.e., with higher photon energy resolution.

Furthermore, we want to point out that the dichroism in Fig. 4 goes to zero below and above the $2 p$ edge without subtracting any offset. Therefore we assume that the systematic errors are smaller than the statistical errors. A repetition of the whole scan over the $2 p$ edge with the opposite helicity of the synchrotron light was deemed to be unnecessary.

\section{RESULTS AND DISCUSSION}

Looking at the dichroism, the most prominent feature of the difference curve in Fig. 4 is the change of the sign at 575 eV. Similar dichroism curves are measured in solid-state studies of Cr films and compounds [17-19]. A direct comparison with the MCD dichroism in a solid-state measurement is shown in Fig. 5. 
circular dichroism in total ion yield

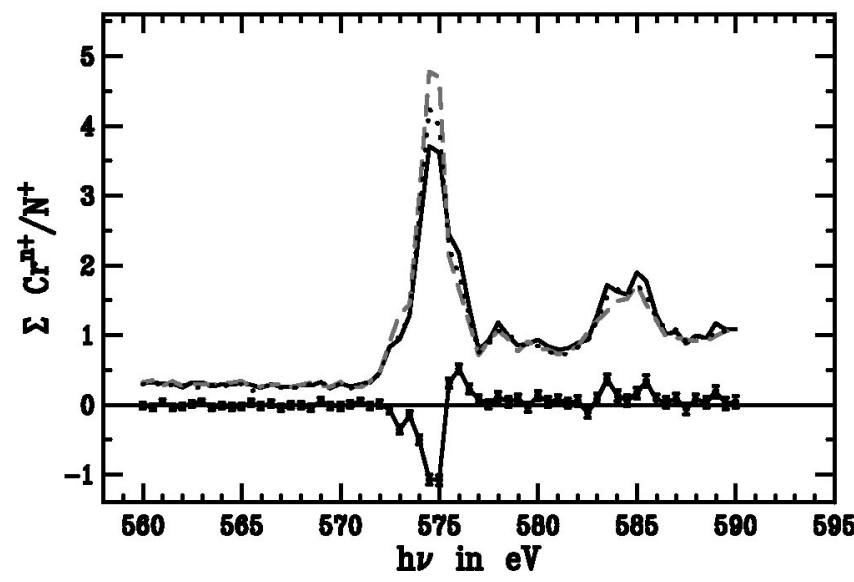

FIG. 4. MCD in the total ion yield of chromium atoms. The dotted line belongs to the count rate for unpolarized atoms, the solid and the broken lines belong to the ion count rate with opposite target polarization. The lower curve shows the difference of the two count rates, i.e., the MCD.

The change of sign can only be explained in a model that takes into account the coupling of the $2 p$ hole with the $3 d$ and $4 s$ shells. The need for including this coupling in the theoretical description was demonstrated for the linear dichroism in the direct $2 p$ photoemission of atomic chromium by Wernet and co-workers [5].

The $2 p$ absorption of chromium atoms can be well described in a single configuration approximation, as has been already pointed out by Arp et al. [20]. Therefore we have performed Hartree-Fock (HF) calculations including relativistic extentions using the COWAN code [21] and the extended Fano theory $[22,23]$, to analyze the MCD absorption spectra. The $2 p$ resonances can be described by an excitation from the Cr ground state $3 d^{5} 4 s^{7} S_{3}$ into intermediate states of the configurations $2 p^{5} 3 d^{6} 4 s$ and $2 p^{5} 3 d^{5} 4 s^{2}$. The $2 p \rightarrow 4 s$ excitation is included in the calculations, however the spectrum
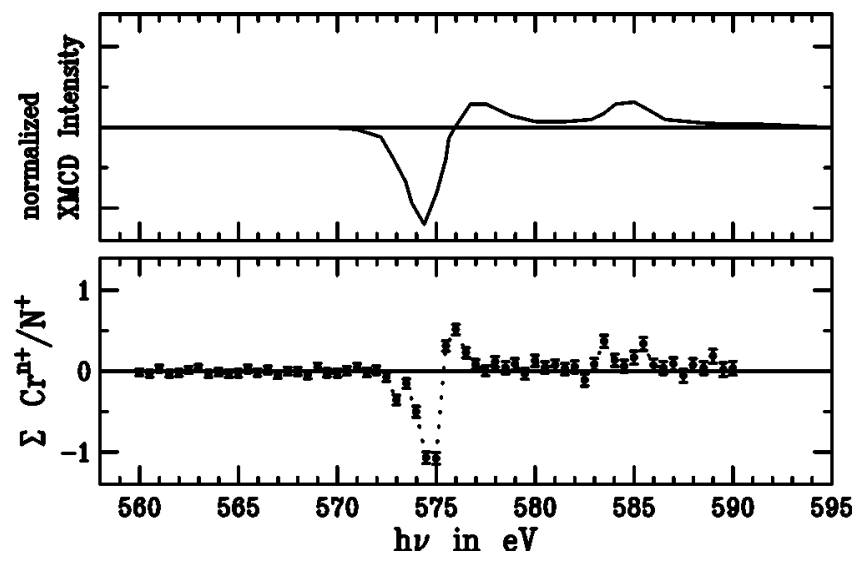

FIG. 5. Comparison with MCD in the solid state. The data from Ref. [19] (upper curve) were shifted to match the position of the MCD found in the gas phase (lower curve). The characteristic sign change at $575.5 \mathrm{eV}$ appears both in the solid state and in the gas phase.

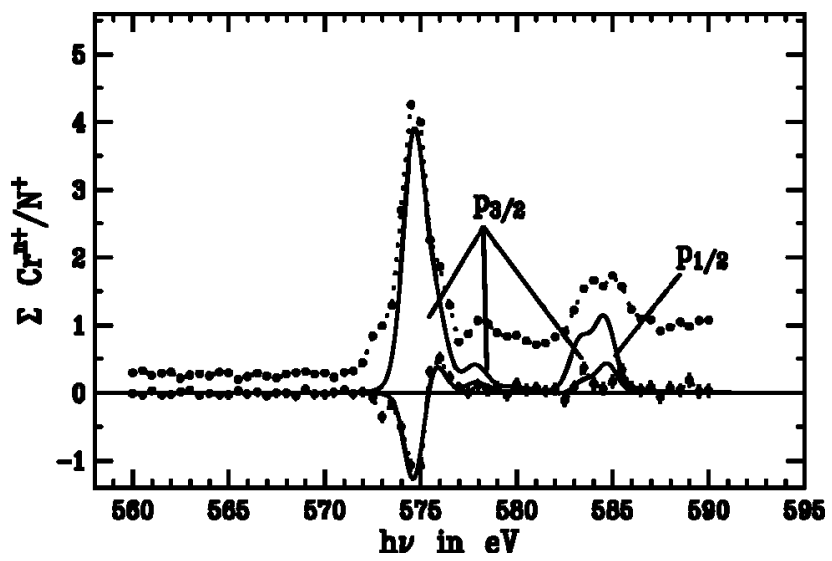

FIG. 6. MCD in the total ion yield of chromium atoms. The broken upper curve shows the measured total ion yield. The solid curves are the results of a HF calculation. The lower curves show the corresponding results for the MCD. The theoretical results have been shifted by $1.6 \mathrm{eV}$ and convoluted with a $700-\mathrm{meV}$ Gaussian peak to include the monochromator bandpass.

is dominated by the $2 p \rightarrow 3 d$ excitation. These resonances will decay mainly due to the $3 p^{-2}$ and $3 d^{-2}$ Auger channels. The resonant photoemission of polarized atoms can be described by the theory developed by Baier, GrumGrzhimailo, and Kabachnik [16]. The differential cross section is given by

$$
\frac{d \sigma}{d \Omega}=\frac{\sigma_{i s o}}{4 \pi}\left(1+\sum_{k_{0} k k_{\gamma}} A_{k_{0} 0} \beta_{k_{0} k k_{\gamma}} F_{k_{0} k k_{\gamma}}\right),
$$

where $A_{k_{0} 0}$ describes the polarization of the ground state, $F_{k_{0} k_{\gamma}}$ depends on the experimental geometry, and $\beta_{k_{0} k_{\gamma}}$ are the generalized $\beta$ parameters [16]. $\sigma_{\text {iso }}$ is the isotropic cross section. The MCD (1) is given by

$$
\beta_{M C D}=\frac{\sum_{n} D^{(n)} \beta_{101}^{(n)}}{\sum_{n} D^{(n)}},
$$

where $n$ runs over all decay channels and $D^{(n)}$ is the partial cross section for channel $n$. The atomic orientation $A_{10}$ is obtained from the rate equation simulations described above. This theoretical approach only describes the resonant part of excitation process, so the direct $2 p$ continuum contribution to the signal is not included. In this calculation the contributions from $p_{1 / 2}$ and $p_{3 / 2}$ excitations can be separated. Figure 6 shows the comparison of the measured and calculated ion yield and dichroism. The dichroism agrees best with the experimental data if we assume an atomic polarization of $40 \%$. The degree of polarization of the light is known to be $\left|S_{3}\right| \simeq 0.8$.

The excitations that contribute to the intensity around 575 $\mathrm{eV}$ contain mostly contributions from the $p_{3 / 2}$ hole. The situation is more difficult for the peaks around $585 \mathrm{eV}$. This region contains significant contribution from $2 p_{1 / 2}$ and $2 p_{3 / 2}$ states. Therefore Eq. (3) cannot be applied in this case. The 
total and partial ion yield of $\mathrm{Cr}$
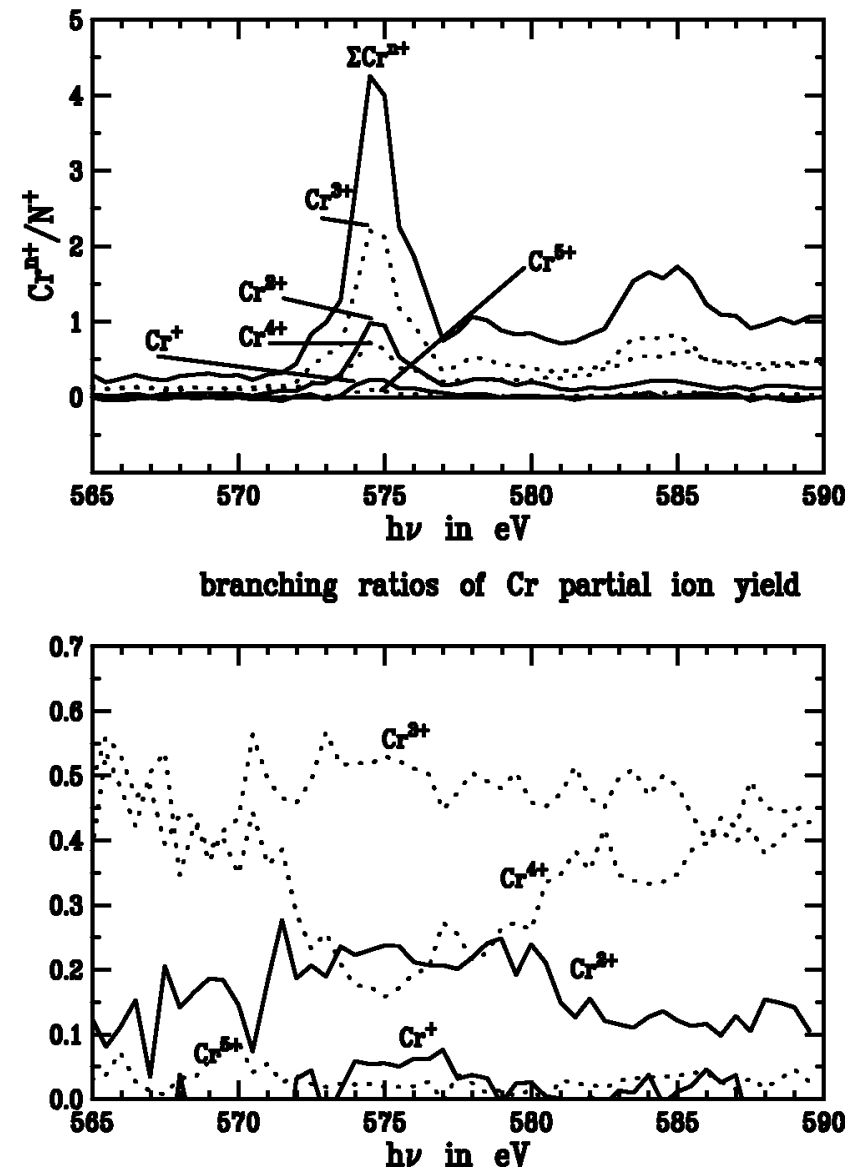

FIG. 7. Total and partial ion yield of unpolarized chromium atoms at the $2 p$ edge. The upper picture shows the ion signals normalized to the $\mathrm{N}^{+}$signal. The lower picture shows the corresponding branching ratios.

contribution of $2 p_{1 / 2}$ and $2 p_{3 / 2}$ states can never be separated by an appropriate choice of the integration regions. A direct proof of the $2 p_{3 / 2}$ contribution to the high-energy half of the $\mathrm{Cr}$ spectrum can be found in the experimental data for the linear alignment dichroism (LAD). As the $2 p_{1 / 2}$ state does not have any alignment, the corresponding excitations cannot contribute to the LAD. In contrast to that, the $\mathrm{Cr} 2 p$ spectrum in Ref. [24] of a $\mathrm{Cr} / \mathrm{Fe}$ layer system shows magnetic linear dichroism in the region around $585 \mathrm{eV}$.

As shown in Fig. 7 the charge states $\mathrm{Cr}^{2+}, \mathrm{Cr}^{3+}$, and $\mathrm{Cr}^{4+}$ have the highest intensity at the $2 p$ edge. The branching ratios are not constant over the $2 p$ edge. The form of the $\mathrm{Cr}^{3+}$ signal more or less resembles the form of the total ion yield. The $\mathrm{Cr}^{2+}$ and $\mathrm{Cr}^{4+}$ signals behave differently. The $\mathrm{Cr}^{2+}$ signal gets a higher branching ratio at the first peak of the total ion yield at $575 \mathrm{eV}$ and less at the second peak at $585 \mathrm{eV}$. For the $\mathrm{Cr}^{4+}$ signal it is just the opposite.

Despite the differences in the branching ratios the MCD asymmetry looks very similar for all three states. This is shown in Fig. 8. The asymmetry for all individual charge states $\mathrm{Cr}^{2+}, \mathrm{Cr}^{3+}$, and $\mathrm{Cr}^{4+}$ resembles the asymmetry of the total ion yield. This indicates that the two-step picture is a good approximation. In the first step, the photon is absorbed

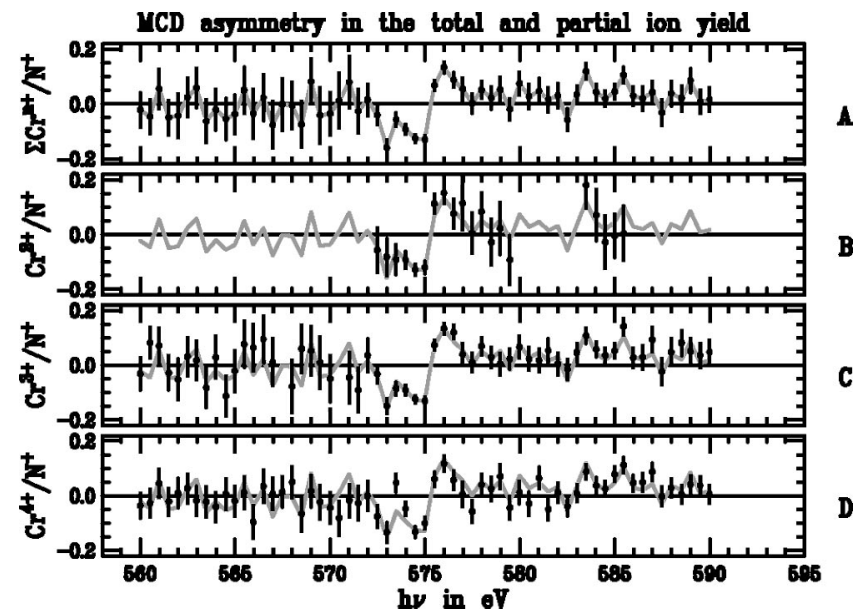

FIG. 8. MCD asymmetry in the total and partial ion yield of chromium atoms. Plot $A$ shows the asymmetry of the total ion yield. The dots and error bars in the plots $B, C$, and $D$ belong to the MCD asymmetry for the $\mathrm{Cr}^{2+}, \mathrm{Cr}^{3+}$, and $\mathrm{Cr}^{4+}$ signals, respectively. Only values with error bars smaller than 0.1 are shown. The gray line is always the asymmetry of the total ion yield.

and the atom is excited. The photon energy selects different excited states with different decay probabilities into differently charged ions. The MCD does only depend on the first step and so all ionic charge states show very similar MCD curves.

With reference to Eq. (2) we define the quantity $A$ which will be called the integral asymmetry:

$$
A=\frac{\sum_{560 \mathrm{eV}-590 \mathrm{eV}}\left(I_{\uparrow \uparrow}-I_{\downarrow \uparrow}\right)}{\sum_{560 \mathrm{eV}-590 \mathrm{eV}}\left(I_{\uparrow \uparrow}+I_{\downarrow \uparrow}\right)} .
$$

The results for $A$ are summarized in Table I. The result compares with zero for the total ion yield as expected from Eq. (2). The changes in the branching ratio over the $2 p$ edge can cause a deviation from this rule for the differently charged ions, as the weight of the $p_{3 / 2}$ and $p_{1 / 2}$ contributions is different. However we were not able to detect a significant variation from zero for any particular charge state. This is an important result for total-ion-yield measurements in which the signal of the $\mathrm{Cr}^{n+}$ ions contribute with a weight factor of $n$ to the measured current. Strictly speaking Eq. (2) is only valid for weight factors of one.

TABLE I. The values of the integral asymmetry $A$ for the total and partial ion yield. The sum extends over all charge states ( $n$ $=1$ to 5 ).

\begin{tabular}{lr}
\hline \hline & \multicolumn{1}{c}{$A(\%)$} \\
\hline$\Sigma \mathrm{Cr}^{n+}$ & $-0.74 \pm 0.48$ \\
$\mathrm{Cr}^{2+}$ & $-0.93 \pm 1.26$ \\
$\mathrm{Cr}^{3+}$ & $-0.41 \pm 0.45$ \\
$\mathrm{Cr}^{4+}$ & $0.30 \pm 0.47$ \\
\hline \hline
\end{tabular}


TABLE II. The values of the MCD asymmetry for the the total ion yield above the $2 p$ edge.

\begin{tabular}{lc}
\hline \hline$h \nu(\mathrm{eV})$ & Asymmetry $(\%)$ \\
\hline 590 & $-0.41 \pm 1.88$ \\
595 & $-0.70 \pm 0.89$ \\
600 & $-0.42 \pm 1.02$ \\
620 & $-0.29 \pm 0.97$ \\
\hline \hline
\end{tabular}

Finally we measured the MCD asymmetry in the total ion yield at energies above the $2 p$ edge. The result is summarized in Table II. All values are compatible with zero as expected in a nonrelativistic model [10]. This result supports the thesis that nonresonant MCD effects are limited to atoms with nonzero orbital angular momentum [9].

\section{SUMMARY}

We showed the results for magnetic dichroism in the total ion yield of gas phase chromium atoms at and above the $2 p$ edge. It was shown that the coupling of the $2 p$ hole with the open $3 d$ and $4 s$ shells is particularly important to understand the structure of the MCD at the $2 p$ edge. Unlike the results found for iron and cobalt in thin films, the MCD at the $2 p$ edge cannot be separated into two distinct $L_{2}$ and $L_{3}$ parts.

Despite some differences of our MCD results with solidstate MCD curves, we assume that a mixing of the $2 p_{1 / 2}$ and $2 p_{3 / 2}$ excitations is also possible in the solid state. Our results show that a double peak structure of the absorbtion signal is no clear indication for a separation of $2 p_{1 / 2}$ and $2 p_{3 / 2}$ excitations.

The two-step model gives a good description of the data for the differently charged ions. The process can be treated as an absorption and inner-shell excitation followed by Auger processes.

At photon energies above the $2 p$ edge no MCD was found, which is consistent with a nonrelativistic description of the photoionization of an atom in an $S$ state.

\section{ACKNOWLEDGMENTS}

This work was supported by Deutsche Forschungsgemeinschafft Grant No. Zi 183/12-1 and by the European Union for beam time at BESSY II.
[1] T. Dohrmann, A. von dem Borne, A. Verweyen, B. Sonntag, M. Wedowski, K. Godehusen, and P. Zimmermann, J. Phys. B 29, 5699 (1996).

[2] A. von dem Borne, T. Dohrmann, A. Verweyen, B. Sonntag, K. Godehusen, and P. Zimmermann, Phys. Rev. Lett. 78, 4019 (1997).

[3] A. von dem Borne, T. Dohrmann, A. Verweyen, B. Sonntag, K. Godehusen, P. Zimmermann, and N.M. Kabachnik, J. Phys. B 31, L41 (1998).

[4] P. Wernet, J. Schulz, B. Sonntag, K. Godehusen, P. Zimmermann, A.N. Grum-Grzhimailo, N.M. Kabachnik, and M. Martins, Phys. Rev. A 64, 042707 (2001).

[5] P. Wernet, J. Schulz, B. Sonntag, K. Godehusen, P. Zimmermann, M. Martins, C. Bethke, and F.U. Hillebrecht, Phys. Rev. B 62, 14331 (2000).

[6] B. Sonntag and P. Zimmermann, Rep. Prog. Phys. 55, 911 (1992).

[7] G. Baum, M.S. Lubell, and W. Raith, Phys. Rev. Lett. 25, 267 (1970)

[8] G. Baum, M.S. Lubell, and W. Raith, Phys. Rev. A 5, 1073 (1972).

[9] G. Prümper, O. Geßner, B. Zimmermann, J. Viefhaus, R. Hentges, H. Kleinpoppen, and U. Becker, J. Phys. B 34, 2707 (2001).

[10] A. Verweyen, A.N. Grum-Grzhimailo, and N.M. Kabachnik, Phys. Rev. A 60, 2076 (1999).

[11] P. Carra, B.T. Thole, M. Altarelli, and X. Wang, Phys. Rev. Lett. 70, 694 (1993).
[12] C.T. Chen, Y.U. Idzerda, H.J. Lin, N.V. Smith, G. Meigs, E. Chaban, G.H. Ho, E. Pellegrin, and F. Sette, Phys. Rev. Lett. 75, 152 (1995).

[13] B.T. Thole, P. Carra, F. Sette, and G. van der Laan, Phys. Rev. Lett. 68, 1943 (1992).

[14] S. Sasaki, K. Miyata, and T. Takada, Jpn. J. Appl. Phys., Part 2 31, L1 794 (1992).

[15] M. Weiss et al., in Scnchrotron Radiation Instrumentation, edited by Piero Pianetta, John Arthur, and Sean Brennan, AIP Conf. Proc. No. 521 (AIP, Melville, NY, 2000).

[16] S. Baier, A. Grum-Grzhimailo, and N. Kabachnik, J. Phys. B 27, 3363 (1994).

[17] T. Böske, W. Clemens, D. Schmitz, J. Kojnok, M. Schäfer, V. Cros, G.Y. Guo, and W. Eberhardt, Appl. Phys. A: Mater. Sci. Process. 61, 119 (1995).

[18] E. Goering, M. Justen, J. Geissler, U. Rüdiger, M. Rabe, G. Güntherodt, and G. Schütz, Appl. Phys. A: Mater. Sci. Process. 74, 747 (2002)

[19] W. O'Brien, B. Tonner, G. Harp, and S. Parkin, J. Appl. Phys. 76, 6462 (1994).

[20] U. Arp, K. Iemura, G. Kutluk, T. Nagata, S. Yagi, and A. Yagishita, J. Phys. B 28, 225 (1995).

[21] R.D. Cowan, The Theory of Atomic Structure and Spectra (University of California Press, Berkeley, 1981).

[22] F. Mies, Phys. Rev. 175, 164 (1968).

[23] M. Martins, J. Phys. B 34, 1321 (2001).

[24] M.M. Schwickert, G.Y. Guo, M.A. Tomaz, W.L. O’Brien, and G.R. Harp, Phys. Rev. B 58, R4 289 (1998). 